\title{
Discovery of a tyrosine-rich sporocyst wall protein in Eimeria tenella
}

Robert A. Walker 1,2, Alisson Niepceron ${ }^{3,4}$, Chandra Ramakrishnan², Laura Sedano 3,4, Adrian B. Hehl², Fabien Brossier ${ }^{3,4^{4}}$ and Nicholas C. Smith ${ }^{1 *}$

\begin{abstract}
Background: Eimeria is an important genus of apicomplexan parasites. A defining feature of these parasites is the oocyst, which is transmitted into the environment via the faeces of definitive hosts. The oocyst wall contains crosslinked, tyrosine-rich proteins and protects eight infectious sporozoites, housed in pairs within a second walled structure, the sporocyst. The biochemical basis for sporocyst wall formation is not known.

Findings: Here, we report the discovery of a novel tyrosine-rich protein, EtSWP1, in Eimeria tenella. Like the tyrosine-rich proteins of the oocyst wall, EtSWP1 is an intrinsically disordered protein with the tyrosine residues concentrated in a specific region of the protein, located immediately following the region of intrinsic disorder. We engineered E. tenella to express mCherry-tagged EtSWP1 and showed that the tagged protein localises specifically to sporocyst walls, indicating that the biochemistry of sporocyst wall assembly is analagous to that of oocyst walls.

Conclusions: Tyrosine-rich proteins are known to be key components of the oocyst wall and we now demonstrate, using gene and protein analyses combined with genetic manipulation, that a novel tyrosine-rich protein is specific for the sporocyst wall. This finding is important because it shows that the biochemistry of these two distinct walls is similar and, hence, brings targeted disruption of sporulation and, therefore, potential neutralisation of oocysts in the environment, a step closer.
\end{abstract}

Keywords: Eimeria, Coccidia, Apicomplexa, Oocyst, Sporocyst, Dityrosine bond

\section{Findings}

Apicomplexan parasites of the genus, Eimeria, cause coccidiosis in a variety of livestock and poultry; in the case of the latter, the industry loses in excess of US $\$ 2$ billion dollars per year due to coccidiosis [1]. These obligate intracellular parasites are highly contagious, due primarily to the resilient nature of the oocyst, an important developmental stage that is transmitted into the environment via the faeces of definitive hosts. The oocyst wall is the primary barrier between the harsh external environment and the infectious cargo, eight dormant sporozoites. However, these sporozoites are further housed in pairs within four sporocysts, a structure that

\footnotetext{
* Correspondence: nicholas.smith@jcu.edu.au

Deceased

${ }^{1}$ Queensland Tropical Health Alliance Research Laboratory, Australian Institute of Tropical Health and Medicine, James Cook University, Cairns Campus, McGregor Road, Smithfield, QLD 4878, Australia

Full list of author information is available at the end of the article
}

is itself characterised by a protective barrier, the sporocyst wall.

Several oocyst wall proteins have been identified and characterised to different degrees [2] including: a cysteine-rich protein, EtOWP6, which is found in the outer layer of the oocyst wall and is related to a family of proteins found in other apicomplexan parasites, namely Cryptosporidium parvum and Toxoplasma gondii; a histidine-rich protein, which localises to the inner oocyst wall; and, GAM56 and GAM82, proteins that are found in wall forming bodies type 2 of macrogametocytes, contain tyrosine-rich regions, and are processed into smaller, tyrosine-rich proteins, which are incorporated into the inner layer of the oocyst wall. GAM56 and GAM82 are also the major components of a transmission blocking vaccine to prevent coccidiosis in poultry [3]. In contrast to oocysts, little is known about the biochemical composition of the sporocyst wall of coccidian parasites [4] despite recent transcriptome profiling of different $E$. tenella developmental stages [5, 6]. 
In order to identify a sporocyst wall-specific protein, we mined the E. tenella genome database using relevant transcript expression data [7] available at www.toxodb.org. Since the sporocyst wall is synthesised only during sporulation, we worked on the assumption that the expression of a sporocyst wall protein would be upregulated specifically in sporulated oocysts. A search for protein coding genes with at least 8-fold increased transcript levels in sporulated oocysts compared to any other stage available (including $2^{\text {nd }}$ generation merozoites, sporozoites and unsporulated oocysts) yielded a total of 17 genes (Fig. 1a). With the notable exception of the sporozoite surface antigen, EtSAG10 (encoded by ETH_00034975), the proteins encoded by these transcripts have no known function or annotation. Expression of ETH_00034975 is known to be upregulated dramatically in the second half of sporulation and maintained in sporozoites and merozoites, indicative of its function as a surface protein on invasive stages of E. tenella [8].

Amongst the 17 genes listed in Fig. 1a, ETH_00000115 stands out; the gene model is predicted to encode a protein that has $7.1 \%$ tyrosine residues (16 of 224 amino acids), which is more than twice that of the next highest (encoded by ETH_00025345). This is potentially significant because, like the oocyst wall, the sporocyst wall of E. tenella fluoresces blue under UV excitation (Fig. 1b,c), a feature that indicates the possible presence of crosslinked tyrosine-rich proteins [9]. Moreover, the predicted amino acid sequence (Fig. 1d) of ETH_00000115 shows that most of these tyrosines are confined to a specific region of the protein, which is analogous to the amino acid sequences of GAM56 and GAM82.

We searched for homologues of ETH_00000115 in other Coccidia but, with the exception of a highly conserved protein in Eimeria necatrix (Fig. 1d), conventional BLASTP analysis against non-redundant protein databases failed to identify homologues of ETH_00000115, including in any other Apicomplexa. Furthermore, ETH_00000115 showed no homology to any previously described protein domains, except for a predicted $\mathrm{N}$-terminal signal peptide, identified using the SignalP 4.1 Server at www.cbs.dtu.dk/ services/SignalP [10]. However, by searching for the KYrich sequence, YKCKKAKGKGKYYKK, we were able to identify a putative homologue in Eimeria brunetti, the otherwise ED-rich region and C-terminal sequence of which are dominated by numerous poly-alanine stretches (Fig. 1d). Like ETH_00000115, the E. necatrix and E. brunetti proteins contain a distinct KY-rich region. All three proteins contain only three cysteine residues but these appear to be strategically placed, with the first near the cleavage site of the signal peptides and the other two at the start of the KY-rich regions, separated by a conserved HGYK sequence.
In both GAM56 and GAM82, the tyrosine-rich regions are also rich in serine residues whereas in ETH_00000115, the tyrosine residues are accompanied by lysine residues. Upstream of, and almost directly adjacent to the lysine and tyrosine-rich (or KY-rich) domain is a 22-residue acidic domain or "ED-rich" domain, which is composed of ten glutamic acid (E) and five aspartic acid (D) residues. Directly downstream of the KY-rich domain are two copies of an imperfect repeat, we have called Repeat 1 (Fig. 1e).

Quantitative reverse-transcriptase PCR (qRT-PCR) was used to assess stage-specific expression of ETH_00000115. The relative transcript abundance of $E T H-00000115$ was determined relative to the et $18 \mathrm{~s}$ small subunit ribosomal RNA for each developmental stage using chicken infection regimens and PCR protocols exactly as described previously [6]. The forward primer was $5^{\prime}$-CGCTGAGGAAGAAATGGAAG-3' and the reverse primer was $5^{\prime}$-TAAGTGCAAAAAGGCC AAGG-3'. Analysis by qRT-PCR confirms that $E T H \_00000115$ transcript is highly abundant in sporulated oocysts but essentially absent in other stages, such as merozoites, gametocytes and unsporulated oocysts (Fig. 1f).

To test the hypothesis that ETH_00000115 is a component of the sporocyst wall, we engineered E. tenella to express an mCherry-tagged version of ETH_00000115. We chose this approach over conventional immunofluorescence to avoid any complications with antibody crossreactivity to other tyrosine-rich proteins present in $E$. tenella. A reporter construct, pETH_00000115-mCherry, was engineered for transfection in E. tenella (Fig. 2a). The reporter plasmid contains the complete coding sequence of ETH_00000115 under the control of its own putative promoter (i.e., within $\sim 1 \mathrm{~kb}$ of sequence upstream of the predicted start codon), in frame with the coding sequence of the fluorescent protein, mCherry, followed by the 3' UTR of etactin. Freshly excysted E. tenella sporozoites were transfected with pETH_00000115-mCherry, reinoculated into naive chickens and oocysts purified 7 days later, as described previously [11]. Microscopic examination of fluorescent oocysts revealed mCherry fluorescence localising specifically to sporocyst walls (Fig. 2b, c). We did not detect mCherry fluorescence in enteric parasite stages such as merozoites and gametocytes. This confirms that ETH_00000115 is a bona fide sporocyst wall protein, which we have therefore named and, from hereon, refer to as "EtSWP1" for Eimeria tenella Sporocyst Wall Protein 1. We observed that mCherry + oocysts could have one, two or four mCherry + sporocyst walls, a result explained by the relatively low efficiency of transfection and the consequent pairing of genetically different gametes, underscored by the high recombination frequency observed during meiosis in Eimeria species [12]. 
a

\begin{tabular}{|l|r|r|r|}
\hline Gene ID & $\begin{array}{c}\text { Fold-change in } \\
\text { sporulated oocysts }\end{array}$ & $\begin{array}{c}\text { Transcript level in } \\
\text { sporulated oocysts } \\
\text { (FPKM) }\end{array}$ & $\begin{array}{c}\text { Number of } \\
\text { tyrosines (\%) }\end{array}$ \\
\hline ETH 00025345 & 72.8 & 37461.6 & $18(3.1)$ \\
\hline ETH_00004255 & 58.3 & 12581.4 & $1(0.2)$ \\
\hline ETH_00039395 & 49.0 & 25538.9 & $0(0.0)$ \\
\hline ETH 00000115 & 36.5 & 708.6 & $16(7.1)$ \\
\hline ETH_00016520 & 23.8 & 912.8 & $1(0.2)$ \\
\hline ETH 00032300 & 23.0 & 5049.8 & $2(0.4)$ \\
\hline ETH 00000120 & 19.0 & 46.1 & $4(3.1)$ \\
\hline ETH 00002890 & 18.4 & 19574.9 & $8(2.0)$ \\
\hline ETH 00034975 & 18.4 & 611.7 & $8(3.1)$ \\
\hline ETH 00030930 & 10.0 & 44429.8 & $1(0.2)$ \\
\hline ETH 00036130 & 9.9 & 17.6 & $0(0.0)$ \\
\hline ETH 00019135 & 9.5 & 120.3 & $9(1.5)$ \\
\hline ETH 00037680 & 8.9 & 91.1 & $1(0.4)$ \\
\hline ETH_00042885 & 8.4 & 19.6 & $5(1.7)$ \\
\hline ETH 00019680 & 8.1 & 101.4 & $6(1.2)$ \\
\hline ETH_00017400 & 8.1 & 17.1 & $6(1.8)$ \\
\hline ETH_00036205 & 8.0 & 142.8 & $0(0.0)$ \\
\hline
\end{tabular}

b

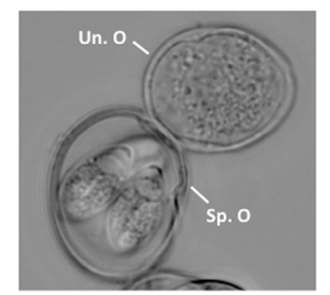

c

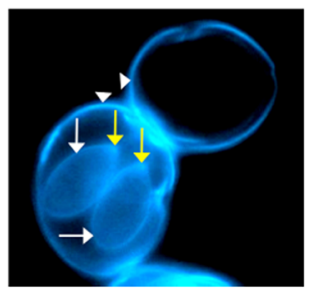

d

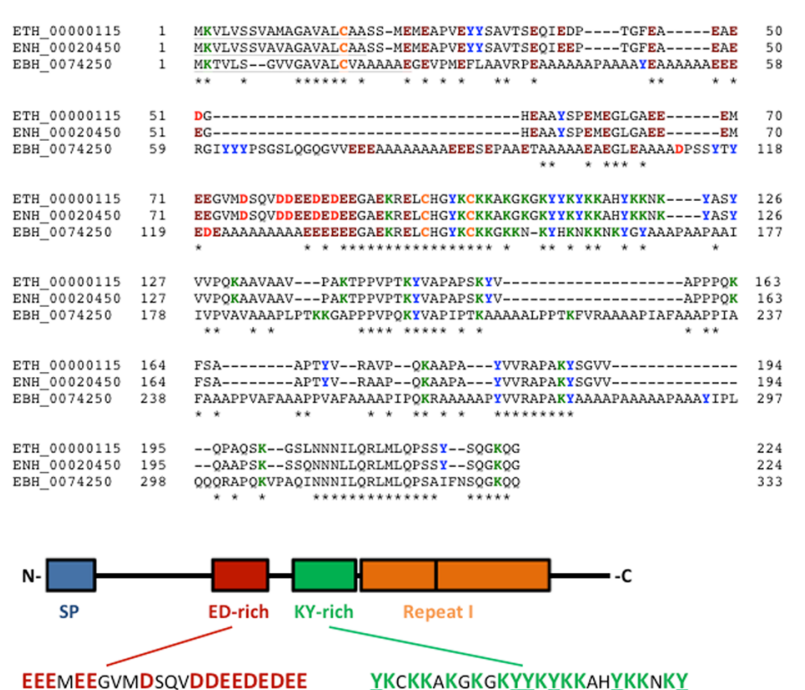

EEEMEEGVMDSQVDDEEDEDEE YKKCKKAKGKGKYYYKYKKAHYKKNKY

ETH_O0000115

f

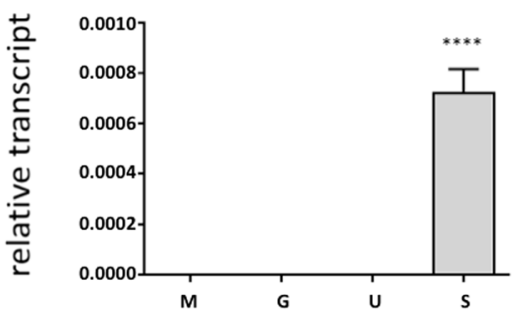

Fig. 1 Identification of a tyrosine-rich protein transcribed specifically in sporulated oocysts of Eimeria tenella. a An integrative search for E. tenella genes with increased expression during sporulation was carried out using the New Search option available at www.toxodb.org, following the path New Search > Search for Genes > Transcript Expression > RNA Seq Evidence. The data set "Life Cycle Stage Transcriptomes (Reid)" was selected for E. tenella strain Houghton, using the FC (fold-change) option. The search identified 17 protein-coding genes that are upregulated with a fold change of $\geq 8$ in the sporulated oocyst sample compared with the maximum expression value recorded for any of the reference samples, including unsporulated oocyst, sporozoite and $2^{\text {nd }}$ generation merozoite. $\mathbf{b}$ Brightfield and $\mathbf{c}$ autofluorescence (UV excitation wavelength $=385 \mathrm{~nm}$ ) microscopy of a bleached unsporulated (Un. O; top right) and a bleached sporulated (Sp. O; bottom left) oocyst of $E$. tenella indicates the possible presence of dityrosine bonds in the inner wall (bleaching removes the outer wall) of both the unsporulated and sporulated oocyst (white arrow-heads) and in the sporocyst walls (white arrows), including the stieda bodies (yellow arrows). Microscopy was done on a Zeiss Axiovert 200 microscope equipped with the Apotome imaging system. Images were generated and analysed using the Axiovision Software (Carl Zeiss SA). d The identification of potential ETH_00000115 (NCBI Reference Sequence: XP_013233236.1) homologues was carried out using BLASTP on the non-redundant NCBI database or on www.toxodb.org and alignments generated using the CLUSTAL O (1.2.1) multiple sequence alignment tool at http://www.ebi.ac.uk/Tools/msa/clustalo/. Conventional BLASTP analysis revealed only a single, highly conserved protein (96 \% identity with ETH_00000115) in Eimeria necatrix (ENH_00020450, NCBI Reference Sequence: XP_013439901.1); however, searching for the KY-rich sequence, YKCKKAKGKGKYYKK, uncovered a further putative homologue in Eimeria brunetti (EBH_0074250, GenBank Reference Sequence: CDJ54052.1; 48 \% identity with ETH_00000115), the ED-rich region and extended C-terminal of which are interspersed with several poly-alanine (A) sequences. The putative signal peptides for the three proteins are underlined. Residues of aspartic acid are highlighted by D. glutamic acid by $\mathbf{E}$, lysine by $\mathbf{K}$, tyrosine by $\mathbf{Y}$ and cysteine by $\mathbf{C}$. Amino acid residues that are conserved across all three species are indicated by *. e A graphic depiction of the predicted ETH_00000115 protein highlighting a lysine (K) and tyrosine (Y)-rich region flanked by a negatively-charged, aspartic acid (D) and glutamic acid (E)-rich region, and a weak repeat sequence. $\mathbf{f}$ Quantitative reverse-transcriptase PCR carried out on different developmental stages of E. tenella [6] confirms the sporulated oocyst-specific expression of ETH_00000115. The relative transcript abundance of ETH-00000115 was determined relative to the et18s small subunit ribosomal RNA for each developmental stage $(\mathrm{M}=$ merozoites, $\mathrm{G}=$ gametocytes, $\mathrm{U}=$ unsporulated oocysts, $\mathrm{S}=$ sporulated oocysts). *** Indicates a statistically significant difference for sporulated oocysts vs every other stage at $P<0.001$ ( $n=3$ samples per developmental stage; one-way ANOVA and Bonferroni multiple comparison posthoc tests using GraphPad Prism Version 6.03, GraphPad Software Inc., USA)

The tyrosine-rich oocyst wall protein, GAM56 is known to possess significant regions of intrinsic disorder within its structure, which is dominated by random coils, with some helices but few sheets/strands [13]. Two repercussions of this are predicted [13]: first, intrinsically disordered proteins are susceptible to proteolytic 


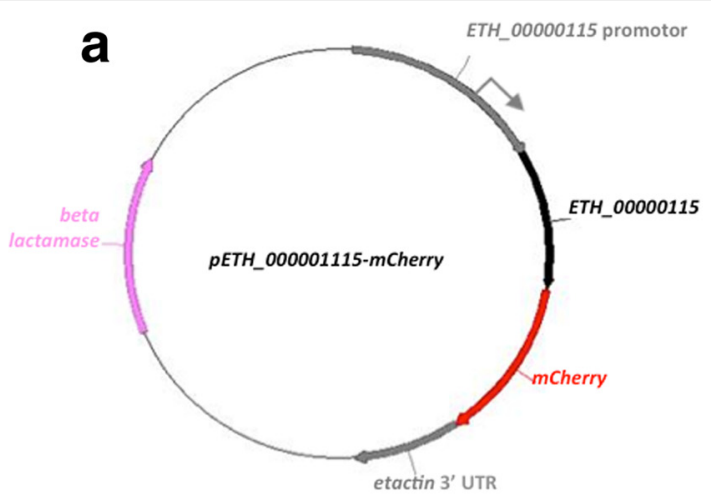

b

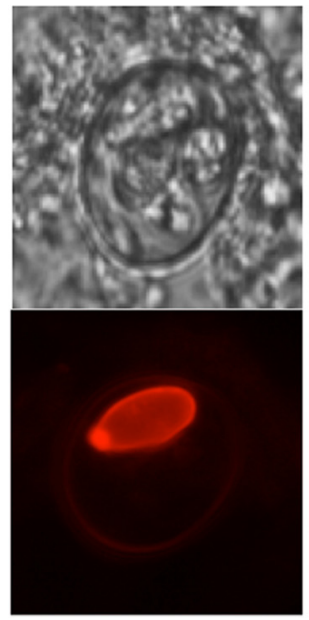

C

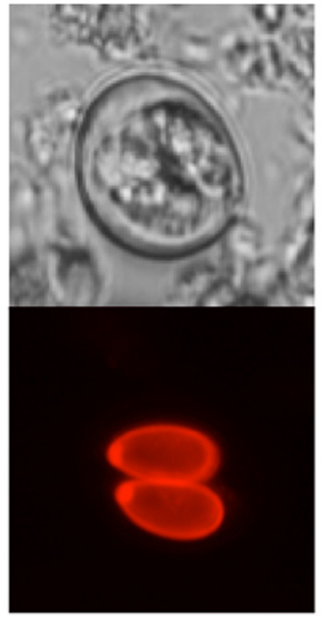

d

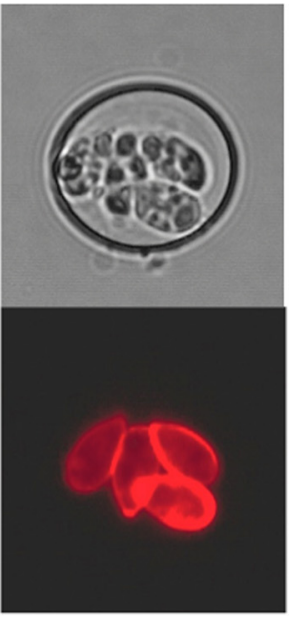

Fig. 2 The tyrosine-rich protein encoded by ETH_00000115 is a sporocyst wall specific protein in Eimeria tenella. a In order to determine the localisation of the tyrosine-rich protein encoded by ETH_00000115 within sporulated oocysts, a reporter plasmid pETH_00000115-mCherry was engineered using the mCherry core construct-1 as a parental plasmid. As there is no predicted intron for ETH_00000115, the putative promoter (982 bp upstream of the predicted start codon) and coding sequence of ETH_00000115 could be PCR amplified from E. tenella genomic DNA as one contiguous product (136,055 to 134,402 from supercontig HG675721) using the forward primer, Mlul-ETH_00000115_F (5'-GGGGATTTITGGG ATGG-3'), and the reverse primer, Sall-ETH_00000115_R (5'-GCAGGGCAAGCAAGGC-3'). The PCR product was cloned into the mCherry core construct-1 via Mlul and Sall, replacing the etmic1 promoter and allowing read-through from the ETH_00000115 coding sequence to the $m$ Cherry coding sequence. Amplification of DNA for cloning was carried out by polymerase chain reaction using Pfu DNA Polymerase (Thermo Scientific) according to the manufacturer's instructions. Transfection of E. tenella sporozoites was carried out as described previously [1 1]. $\mathbf{b}, \mathbf{c}, \mathbf{d}$ Brightfield (upper panels) and red fluorescence (wavelength $=590 \mathrm{~nm}$, lower panels) microscopy of pETH_00000115-mCherry sporulated oocysts of E. tenella confirms that the tyrosine-rich protein encoded by ETH_00000115 is expressed specifically in the sporocyst wall. Recombination resulting from pairing of transfected and non-transfected gametes means that one, two or four sporocysts within any single oocyst may display the mCherry signal; $\mathbf{b}$ shows a single sporocyst of four, $\mathbf{c}$ shows two sporocysts of four and $\mathbf{d}$ shows all four sporocysts exhibiting fluorescence. Microscopy was done on a Zeiss Axiovert 200 microscope equipped with the Apotome imaging system. Images were generated and analysed using the Axiovision Software (Carl Zeiss SA)

cleavage, as observed for GAM56; and, second, the inherent flexibility of disordered proteins may increase the possibility of tyrosine residues from individual tyrosinerich proteins coming into the proximity of each other more readily, thereby facilitating crosslinking. We used DISOPRED3 [14] to analyse the amino acid sequences of EtSWP1, EnSWP1 and EbSWP1 to determine if these proteins possess similar secondary structures to GAM56; DISOPRED3 has been used recently, with significant success, to identify regions of intrinsic disorder in the structure of apicomplexan proteins [15]. We found that all three SWP1s were dominated by coil structures, with some helices and very little sheet/ strand structures (Additional file 1: Figure S1). Analyses using I-Tasser [16-19] generated similar predictions (Additional file 2: Figure S2). DISOPRED3 also predicted significant regions of intrinsic disorder within all the SWP1s, most notably in the ED-rich region preceding the KY-rich sequence (Additional file 3: Figure S3); GAM56 is, likewise, intrinsically 
disordered just prior to the tyrosine-rich region. We therefore predict that SWP1 behaves similarly to GAM56 in wall assembly.

BLASTP searches also failed to identify any homologues of EtSWP1 in the cyst forming coccidians, Toxoplasma gondii, Neospora caninum or Hammondia hammondi, but, by searching for proteins with similar percentages of lysine, tyrosine, glutamic acid and aspartic acid, three sequences (TGME49_037080, NCLIV_050960 and HHA_237080) that are highly conserved across these three parasites were discovered (each with $\sim 21 \%$ identity with EtSWP1). However, TGME49_037080 has already been identified as more likely to be a component of the oocyst wall and not the sporocyst wall of $T$. gondii by transcriptomic [20] and proteomic [21] analyses.

There are other tyrosine-rich proteins indicated by transcriptomic analysis of $T$. gondii oocysts as being potential components of the sporocyst wall [20] but the localisation of these proteins specifically to the sporocyst wall has not been confirmed. The discovery that EtSWP1 is targeted specifically to the sporocyst wall is important because, in combination with the recent finding that the sporocyst wall contains cysteine-rich proteins [22], it shows that the construction of the sporocyst wall is biochemically similar to the oocyst wall despite being assembled via a different cell biology mechanism; in contrast to oocyst wall formation, which occurs via sequential, coordinated migration and disaggregation of veil forming bodies, wall forming bodies type 1 and wall forming bodies type 2 [23], the wall of the sporocyst forms via condensation of cytoplasmic material not contained in defined organelles or vesicles $[22,24]$. Our discovery of a tyrosine-rich protein in the sporocyst wall brings targeted disruption of sporulation and, therefore, potential neutralisation of oocysts in the poultry house, a step closer.

\section{Ethics statement}

Animal experimentation was conducted under protocols approved by the University of Technology, Sydney Animal Ethics Committee (protocol numbers 2008-96 and 2008-188) under a protocol (registration number 2012-11-9) and/or by the ethics committee, CEEA VdL, and according to French legislation (French Government Decree 2001-464) and EEC regulations (86/609/CEE).

\section{Additional files}

Additional file 1: Figure S1. Secondary structure predictions for SWP1 from (a) Eimeria tenella, (b) Eimeria necatrix and (c) Eimeria brunetti, determined using DISOPRED3 (http://bioinf.cs.ucl.ac.uk/disopred) indicating that all three proteins are dominated by random coils ( $71 \%$, $71 \%$ and $73 \%$, respectively), with significant helices $(25 \%, 25 \%$ and $27 \%$, respectively) but few sheet/strand structures (4\%, 4\% and $0 \%$, respectively). (TIFF $1521 \mathrm{~kb}$ )
Additional file 2: Figure S2. Secondary structure predictions for SWP1 from (a) Eimeria tenella, (b) Eimeria necatrix and (c) Eimeria brunetti, determined using I-Tasser (http://zhanglab.ccmb.med.umich.edu/lTASSER/). red $\mathrm{H}=$ helix, blue $\mathrm{S}=$ strand, $\mathrm{C}=$ coil. The lysine/tyrosine-rich region is highlighted in green and the glutamic acid/aspartic acid-rich region in yellow. (TIFF 2702 kb)

Additional file 3: Figure S3. Identification of predicted intrinsically disordered structures in SWP1 from (a) Eimeria tenella, (b) Eimeria necatrix and (c) Eimeria brunetti, determined using DISOPRED3 (http:// bioinf.cs.ucl.ac.uk/disopred). (TIFF 2702 kb)

\section{Competing interests}

The authors declare that they have no competing interests.

\section{Authors' contributions}

RAW, AN, CR, LS and FB carried out the experimental work. RAW, AN, CR, $A B H$ and NCS analysed and interpreted the data. RAW and FB conceived and designed the study. RAW and NCS wrote the manuscript. RAW, AN, CR, LS, $\mathrm{ABH}$ and NCS reviewed drafts of the manuscript and approved the final draft for publication.

\section{Acknowledgements}

We are grateful to the staff of the Ernst Facility at the University of Technology, Sydney, for their care of the chickens used in this study. NCS gratefully acknowledges funding provided by the Australian Research Council for Discovery Project DP0664013. NCS and ABH are thankful for funding from Bellberry Limited. NCS and FB were recipients of an Australian Government Department of Innovation, Industry, Science and Research International Science Linkage grant (FR110089). RAW was supported by a Swiss Government Excellence Postdoctoral Scholarship from the Swiss Confederation. None of the funding bodies played any role in: the design, collection, analysis, or interpretation of data; the writing of the manuscript; or the decision to submit the manuscript for publication.

\section{Author details}

${ }^{1}$ Queensland Tropical Health Alliance Research Laboratory, Australian Institute of Tropical Health and Medicine, James Cook University, Cairns Campus, McGregor Road, Smithfield, QLD 4878, Australia. ${ }^{2}$ Institute of Parasitology, University of Zurich, Winterthurerstrasse 266a, CH-8057 Zurich, Switzerland. ${ }^{3}$ INRA, UMR1282, Infectiologie et Santé Publique, Laboratoire Apicomplexes et Immunité Mucosale, F-37380 Nouzilly, France. ${ }^{4}$ Université François-Rabelais de Tours, UMR1282 Infectiologie et Santé Publique, F-37000 Tours, France.

Received: 4 January 2016 Accepted: 24 February 2016

Published online: 02 March 2016

\section{References}

1. Shirley MW, Smith AL, Tomley FM. The biology of avian Eimeria with an emphasis on their control by vaccination. Adv Parasitol. 2005;60:285-330.

2. Walker RA, Ferguson DJ, Miller CM, Smith NC. Sex and Eimeria: a molecular perspective. Parasitology. 2013;140:1701-17.

3. Sharman PA, Smith NC, Wallach MG, Katrib M. Chasing the golden egg: vaccination against poultry coccidiosis. Parasite Immunol. 2010;32:590-8.

4. Samuelson J, Bushkin GG, Chatterjee A, Robbins PW. Strategies to discover the structural components of cyst and oocyst walls. Eukaryot Cell. 2013;12: 1578-87.

5. Novaes J, Rangel LT, Ferro M, Abe RY, Manha AP, de Mello JC, Varuzza L, Durham AM, Madeira AM, Gruber A. A comparative transcriptome analysis reveals expression profiles conserved across three Eimeria spp. of domestic fowl and associated with multiple developmental stages. Int J Parasitol. 2012;42:39-48.

6. Walker RA, Sharman PA, Miller CM, Lippuner C, Okoniewski M, Eichenberger R, Ramakrishnan C, Brossier F, Deplazes P, Hehl AB, Smith NC. RNA Seq analysis of the Eimeria tenella gametocyte transcriptome reveals clues about the molecular basis for sexual reproduction and oocyst biogenesis. BMC Genomics. 2015;16:94.

7. Reid AJ, Blake DP, Ansari HR, Billington K, Browne HP, Bryant J, Dunn M, Hung SS, Kawahara F, Miranda-Saavedra D, Malas TB, Mourier T, Naghra H, Nair M, Otto TD, Rawlings ND, Rivailler P, Sanchez-Flores A, Sanders M, Subramaniam C, Tay YL, Woo Y, Wu X, Barrell B, Dear PH, Doerig C, Gruber 
A, Ivens AC, Parkinson J, Rajandream MA, Shirley MW, Wan KL, Berriman M, Tomley FM, Pain A. Genomic analysis of the causative agents of coccidiosis in domestic chickens. Genome Res. 2014;24:1676-85.

8. Tabares E, Ferguson D, Clark J, Soon P-E, Wan K-L, Tomley F. Eimeria tenella sporozoites and merozoites differentially express glycosylphosphotidylinositol-anchored variant surface proteins. Mol Biochem Parasitol. 2004;135:123-32.

9. Belli SI, Wallach MG, Luxford C, Davies MJ, Smith NC. Roles of tyrosine-rich precursor glycoproteins and dityrosine- and 3,4-dihydroxyphenylalaninemediated protein cross-linking in development of the oocyst wall in the coccidian parasite Eimeria maxima. Eukaryot Cell. 2003;2:456-64.

10. Petersen TN, Brunak S, von Heijne G, Nielsen H. SignalP 4.0: discriminating signal peptides from transmembrane regions. Nat Methods. 2011:8:785-6.

11. Rieux A, Gras S, Lecaille F, Niepceron A, Katrib M, Smith NC, Lalmanach G, Brossier F. Eimeripain, a cathepsin B-like cysteine protease, expressed throughout sporulation of the apicomplexan parasite Eimeria tenella. PLoS One. 2012;7, e31914.

12. Kurth $M$, Entzeroth R. Reporter gene expression in cell culture stages and oocysts of Eimeria nieschulzi (Coccidia, Apicomplexa). Parasitol Res. 2009;104 303-10.

13. Mai K, Smith NC, Feng ZP, Katrib M, Slapeta J, Slapetova I, Wallach MG, Luxford C, Davies MJ, Zhang X, Norton RS, Belli SI. Peroxidase catalysed cross-linking of an intrinsically unstructured protein via dityrosine bonds in the oocyst wall of the apicomplexan parasite, Eimeria maxima. Int J Parasitol. 2011;41:1157-64.

14. Jones DT, Cozzetto D. DISOPRED3: precise disordred region predictions with annotated protein-biding activity. Bioinformatics. 2015;31:857-63.

15. Guy AJ, Irani V, MacRaild CA, Anders RF, Norton RS, Beeson JG, Richards JS, Ramsland PA. Insights into the immunological properties of intrinsically disordered malaria proteins using proteome scale predictions. PLoS One. 2015;10, e0141729.

16. Zhang Y. I-TASSER server for protein $3 D$ structure prediction. BMC Bioinformatics. 2008;9:40

17. Roy A, Kucukural A, Zhang Y. I-TASSER: a unified platform for automated protein structure and function prediction. Nat Protoc. 2010;5:725-38.

18. Yang J, Yan R, Roy A, Xu D, Poisson J, Zhang Y. The I-TASSER Suite: protein structure and function prediction. Nat Methods. 2015;12:7-8.

19. Yang J, Zhang Y. I-TASSER server: new development for protein structure and function predictions. Nucleic Acids Res. 2015:43:W174-81

20. Fritz HM, Buchholz KR, Chen X, Durbin-Johnson B, Rocke DM, Conrad PA, Boothroyd JC. Transcriptomic analysis of Toxoplasma development reveals many novel functions and structures specific to sporozoites and oocysts. PLoS One. 2012;7, e29998.

21. Fritz HM, Bowyer PW, Bogyo M, Conrad PA, Boothroyd JC. Proteomic analysis of fractionated Toxoplasma oocysts reveals clues to their environmental resistance. PLoS One. 2012;7, e29955.

22. Jonscher E, Erdbeer A, Gunther M, Kurth M. Two COWP-like cysteine rich proteins from Eimeria nieshulzi (Coccidia, Apicomplexa) are expressed during sporulation and involved in the sporocyst wall formation. Parasit Vectors. 2015:8:395.

23. Ferguson DJP, Belli SI, Smith NC, Wallach MG. The development of the macrogamete and oocyst wall in Eimeria maxima: immuno-light and electron microscopy. Int J Parasitol. 2003;33:1329-40.

24. Ferguson DJP, Dubremetz J-F. The ultrastructure of Toxoplasma gondii. In: Weiss LM, Kim K, editors. Toxoplasma gondii: the model apicomplexan perspectives and methods. 2nd ed. London, Waltham and San Diego: Academic Press; 2014. p. 20-59.

\section{Submit your next manuscript to BioMed Central and we will help you at every step:}

- We accept pre-submission inquiries

- Our selector tool helps you to find the most relevant journal

- We provide round the clock customer support

- Convenient online submission

- Thorough peer review

- Inclusion in PubMed and all major indexing services

- Maximum visibility for your research

Submit your manuscript at www.biomedcentral.com/submit

) Biomed Central 